“ (C) 2017 IEEE. Personal use of this material is permitted. Permission from IEEE must be obtained for all other uses, in any current or future media, including

reprinting/republishing this material for advertising or promotional purposes, creating new collective works, for resale or redistribution to servers or lists, or reuse of any copyrighted component of this work in other works." 


\title{
Speed Sensorless Model Predictive Current Control with Ability to Start a Free Running Induction Motor
}

\author{
Haitao Yang ${ }^{1, *}$, Yongchang Zhang ${ }^{2,3}$, Paul Walker ${ }^{1}$, Jiejunyi Liang ${ }^{1}$, Nong Zhang ${ }^{1}$, Bo Xia ${ }^{2}$ \\ ${ }^{1}$ School of Electrical, Mechanical, and Mechatronic Systems, University of Technology, Sydney, \\ NSW 2007, Australia \\ ${ }^{2}$ Inverter Technologies Engineering Research Center of Beijing, North China University of Tech- \\ nology, Beijing, 100144, China. \\ ${ }^{3}$ Collaborative Innovation Center of Electric Vehicles in Beijing, Beijing 100081, China. \\ *Haitao.Yang@student.uts.edu.au
}

\begin{abstract}
In this paper, a speed-sensorless finite control set-model predictive current control method is proposed based on an adaptive full order observer. The control system features simplicity and low cost because of no requirement for speed measurement, modulator and tuning of weighting factors. In most sensorless based schemes, the motor is assumed to be started from standstill. There is limited research considering starting a free running motor with unknown rotational direction and speed. To start a free-running induction motor (IM), the feedback gain matrix is designed to guarantee the convergence of estimated speed to actual speed even with incorrect initial value. To improve the efficiency, amplitude of flux is adjusted along with load condition. The presented results show that the proposed method is able to smoothly start an IM with unknown initial speed and work well over a wide speed range. The effectiveness of the proposed method is verified by both simulation and experimental tests on a two-level inverter fed IM drive platform.
\end{abstract}

\section{NOMENCLATURE}

Symbols

$i \quad$ Current Vector

$\boldsymbol{u} \quad$ Voltage Vector

$\psi \quad$ Flux Vector

$L, R \quad$ Inductance and resistance

$\omega \quad$ Angular frequency

Time 


$\begin{array}{ll}j & \text { Imaginary component of a complex variable } \\ s & \text { Laplace operator } \\ \triangle & \text { Difference } \\ e & \text { Error } \\ \tau & \text { Filter time constant } \\ \theta & \text { Angle }\end{array}$

Subscripts

$s, m, r \quad$ Stator, mutual, and rotor

$d, q \quad \mathrm{~d}$-axis and q-axis in rotor flux reference frame

Superscripts

$\begin{array}{ll}\text { ref } & \text { Reference value } \\ \wedge & \text { Estimated value } \\ k & k \text { th instant }\end{array}$

\section{Introduction}

Finite control set-model predictive control (FCS-MPC) has been developed as a viable high performance control method for motor drives and converters. Due to its simplicity in concept, constraint handling and simultaneous control of different objectives, FCS-MPC has attracted much attention recently [1-7]. It is shown that the performance of FCS-MPC is comparable to or better than that of some well established methods, such as field oriented control (FOC) $[2,8]$ and direct torque control (DTC) [9].

Similar to DTC, the available voltage vector is directly selected in FCS-MPC and thus no modulator is required. For FCS-MPC, the best voltage vector is determined by solving an online optimization problem. While for DTC, it is selected from an offline designed heuristic table. The vector selected from FCS-MPC is better in satisfying control objectives since the most suitable one is selected in theory. Additionally, as the future variation of concerned control variables can be predicted in FCS-MPC, the dynamic error can be corrected in advance before it occurs. Due to the effort from many researchers, FCS-MPC has been successfully applied to achieve high performance control of different types of motors and converters [10,11].

Generally, flux amplitude and torque are both controlled in high performance motor drives. Thus, a proper weighting factor is required in conventional predictive torque control (PTC) to achieve simultaneous control of these two variables, because they are different in both unit and 
magnitude [12]. As the weighting factor is usually adjusted based on simulation and experimental tests, it is not suitable for general purpose applications. To address this problem, methods such as ranking based approach [3], online optimization via minimizing torque ripple [13] and model predictive flux control $[14,15]$, etc. have been proposed. However, due to high nonlinearity of FCSMPC and AC motor, the exact relationship between weighting factor and the control performance is still not clear and further research is required.

To obtain the information of unmeasured states in practical application, various observers have been designed and employed in predictive control. In [16], the observer with sliding mode feedback gains is designed based on $\mathrm{H}$-inf method to reduce the impact of parameter variation. In $[17,18]$, improved performance is obtained in five-phase IM drive by incorporating a rotor current observer. The poles of observer are allocated based on Butterworth filter design. In [19], Kalman filter and Luenberger observer are investigated to construct unmeasured rotor variables for achieving better performance. To improve the robustness of the controller, a disturbance observer is employed in [20]. For speed sensorless operation, an inherently encoderless observer is applied in [21] to remove the disturbance produced by inaccurate speed.

In this paper, an adaptive full order observer (AFO) [22,23] is employed to achieve speed sensorless operation of FCS-MPC. Due to the feedback of current estimation error and its adaptability to the variation of motor parameters, AFO has good accuracy over a wide speed range. In the existing literature, AFO are mainly investigated related to stability and robustness against parameter variation. In [24], the feedback gain matrix is designed to stabilize the observer in regenerating mode during low speed operation. The stability of simultaneous estimation of rotor speed and stator resistance is further analyzed in [25]. To obtain desired performance under different operating conditions, a set of feedback gains is adopted in [23]. To address poor accuracy with low sampling frequency and the risk of stability at high speed operation in discrete-time implementation, different discretization methods are studied in $[26,27]$ to replace conventional forward Euler method. However, little research considers starting a free-running motor with unknown rotational direction and speed. When the motor is running at high speed before being powered by the inverter, the estimated speed with zero initial value may fail to converge to actual speed if the feedback gain matrix is not properly designed. The mismatch between actual speed and estimated speed would result in wrong estimation of motor states and precise control of the motor becomes difficult.

Normally, the motor is assumed to be started from standstill. However, for applications with large shaft inertia, it is time consuming to wait a free-running motor to stop. Thus, it would be beneficial for a rotating motor to resume to normal operation after a power interruption, for example. This imposes the requirement for starting a motor with nonzero initial speed. When a position/speed sensor is not equipped, high inrush current may occur, due to large difference between the rotating frequency of the motor and output frequency of the inverter [28]. To start a free running motor without a speed sensor, some methods that investigate current or back electromotive force responses can be found in [28,29]. Some of these methods require proportional-integral (PI) controller to regulate stator current. Some other methods need to inject voltage with variable 
amplitude and phase angle based on a modulator. It is clear that these methods can not be simply used in FCS-MPC due to different way of operation compared with linear controller and the lack of modulator.

To guarantee the successful start of a free-running motor with an AFO, the convergence of the estimated speed is investigated based on the estimation error of stator current. And a feedback gain matrix is designed to ensure the estimated speed can converge to actual speed even if there is a large initial difference. Furthermore, it is incorporated with finite control set-model predictive current control (FCS-MPCC) to achieve high performance operation. The reference generation during the estimation of free-running speed and the shifting to normal operation are well designed. Both simulation and experimental results show that smooth and quick start of a rotating motor is achieved by the proposed FCS-MPCC and satisfactory performance can be obtained over a wide speed range.

In Section 2, mathematical models of IM and AFO are given. Section 3 presents theoretical analysis of speed convergence condition of a rotor flux based AFO. And a new feedback gain matrix is derived to ensure that speed estimation can work properly with any initial rotor speed. In Section 4, detailed implementation of FCS-MPCC with the proposed AFO is introduced. The simulation and experimental results are discussed in Section 5. Finally, this paper is concluded in Section 6.

\section{Mathematical Model of IM and AFO}

\subsection{Dynamic Equations of IM}

Using stator current $\boldsymbol{i}_{s}$ and rotor flux $\boldsymbol{\psi}_{r}$ as state variables, the electromagnetic model of the IM can be expressed in stationary coordinates as follows.

$$
\left\{\begin{array}{l}
s \boldsymbol{i}_{s}=-\frac{1}{T_{\sigma}} \boldsymbol{i}_{s}+\lambda L_{m}\left(\frac{1}{T_{r}}-j \omega_{r}\right) \boldsymbol{\psi}_{r}+\frac{1}{\sigma L_{s}} \boldsymbol{u}_{s} \\
s \boldsymbol{\psi}_{r}=\frac{L_{m}}{T_{r}} \boldsymbol{i}_{s}-\left(\frac{1}{T_{r}}-j \omega_{r}\right) \boldsymbol{\psi}_{r}
\end{array}\right.
$$

where, $\sigma=1-L_{m}^{2} /\left(L_{s} L_{r}\right), \lambda=1 /\left(L_{s} L_{r}-L_{m}^{2}\right), T_{r}=L_{r} / R_{r}, T_{\sigma}=\sigma L_{s} /\left(R_{s}+\left(L_{m} / L_{r}\right)^{2} R_{r}\right)$.

The output torque can be calculated as:

$$
T_{e}=\frac{3}{2} n_{p} \frac{L_{m}}{L_{r}} \boldsymbol{\psi}_{r} \otimes \boldsymbol{i}_{s}
$$

where, $n_{p}$ is the number of pole pairs and $\otimes$ represents cross product.

Despite that better prediction result can be obtained using more accurate discrete-time model [7], simple forward-Euler method is still employed in many papers [30,31], owing to its simplicity and acceptable accuracy when sampling frequency is sufficiently high. Based on forward-Euler 
method, the current and rotor flux can be predicted using the following equations.

$$
\begin{aligned}
\boldsymbol{i}_{s}^{k+1} & =\boldsymbol{i}_{s}^{k}+T_{s c}\left(-\frac{\boldsymbol{i}_{s}^{k}}{T_{\sigma}}+\lambda L_{m}\left(\frac{1}{T_{r}}-j \omega_{r}^{k}\right) \boldsymbol{\psi}_{r}^{k}+\frac{\boldsymbol{u}_{s}^{k}}{\sigma L_{s}}\right) \\
\boldsymbol{\psi}_{r}^{k+1} & =\boldsymbol{\psi}_{r}^{k}+T_{s c}\left(\frac{L_{m}}{T_{r}} \boldsymbol{i}_{s}^{k}-\left(\frac{1}{T_{r}}-j \omega_{r}^{k}\right) \boldsymbol{\psi}_{r}^{k}\right)
\end{aligned}
$$

where, $T_{s c}$ is sampling period.

\subsection{Basic Principle of AFO}

Based on (1), the AFO can be constructed as

$$
\begin{aligned}
s \hat{\boldsymbol{x}} & =\hat{\boldsymbol{A}} \hat{\boldsymbol{x}}+\boldsymbol{B} \boldsymbol{u}_{s}+\boldsymbol{G}\left(\boldsymbol{i}_{s}-\hat{\boldsymbol{i}}_{s}\right) \\
\hat{\boldsymbol{A}} & =\left[\begin{array}{cc}
-\frac{1}{T_{\sigma}} & \lambda L_{m}\left(\frac{1}{T_{r}}-j \hat{\omega}_{r}\right) \\
\frac{L_{m}}{T_{r}} & -\frac{1}{T_{r}}+j \hat{\omega}_{r}
\end{array}\right] \\
\boldsymbol{B} & =\left[\begin{array}{c}
\frac{1}{\sigma L_{s}} \\
0
\end{array}\right] \\
\boldsymbol{G} & =\left[\begin{array}{c}
\boldsymbol{g}_{1} \\
\boldsymbol{g}_{2}
\end{array}\right]=\left[\begin{array}{c}
g_{1 r}+j \cdot g_{1 i} \\
g_{2 r}+j \cdot g_{2 i}
\end{array}\right]
\end{aligned}
$$

where, $\hat{\boldsymbol{x}}=\left[\begin{array}{ll}\hat{\boldsymbol{i}}_{s} & \hat{\boldsymbol{\psi}}_{r}\end{array}\right]^{T}$. The speed can be estimated based on conventional adaptation law as

$$
\begin{aligned}
\frac{d \hat{\omega}_{r}}{d t} & =\left(\boldsymbol{i}_{s}-\hat{\boldsymbol{i}}_{s}\right) \otimes \hat{\psi}_{r} \\
& =\left|\boldsymbol{i}_{s}-\hat{\boldsymbol{i}}_{s}\right| \cdot\left|\hat{\psi}_{r}\right| \sin \theta .
\end{aligned}
$$

where, $\theta$ is the phase angle between complex vector $\hat{\psi}_{r}$ and $\left(\boldsymbol{i}_{s}-\hat{\boldsymbol{i}}_{s}\right)$, namely

$$
\theta=\operatorname{angle}\left(\hat{\psi}_{r}\right)-\operatorname{angle}\left(\boldsymbol{i}_{s}-\hat{\boldsymbol{i}}_{s}\right)
$$

In practical applications, a proportional-integral (PI) controller is generally used to obtain $\hat{\omega}_{r}$ as

$$
\hat{\omega}_{r}=\left(k_{p \omega}+k_{i \omega} / s\right) \cdot\left(\left(\boldsymbol{i}_{s}-\hat{\boldsymbol{i}}_{s}\right) \otimes \hat{\psi}_{r}\right)
$$

For better accuracy in digital implementation, the so-called Heun's method $[32,33]$ is used to discretize (5) and the results are show as follows.

$$
\left\{\begin{array}{l}
\hat{\boldsymbol{x}}_{p}^{k+1}=\hat{\boldsymbol{x}}^{k}+T_{s c}\left(\boldsymbol{A} \hat{\boldsymbol{x}}^{k}+\boldsymbol{B} \boldsymbol{u}_{s}^{k}+\boldsymbol{G}\left(\boldsymbol{i}_{s}-\hat{\boldsymbol{i}}_{s}\right)\right) \\
\hat{\boldsymbol{x}}^{k+1}=\hat{\boldsymbol{x}}_{p}^{k+1}+\frac{T_{s c}}{2} \boldsymbol{A}\left(\hat{\boldsymbol{x}}_{p}^{k+1}-\hat{\boldsymbol{x}}^{k}\right)
\end{array}\right.
$$




\section{Analysis of Speed Convergence Condition}

To ensure $\hat{\omega}_{r}$ always converges to actual speed $\omega_{r}$, the derivative of $\hat{\omega}_{r}$ shown in (9) should change the sign along with $\triangle \omega_{r}=\omega_{r}-\hat{\omega}_{r}$. Namely if (14) holds, $\hat{\omega}_{r}$ will always approach to $\omega_{r}$ when $\triangle \omega_{r}$ is not zero.

$$
\operatorname{sign}\left(\omega_{r}-\hat{\omega}_{r}\right) \cdot \frac{d \hat{\omega}_{r}}{d t}>0
$$

According to (10), (14) can be equivalently rewritten as

$$
\operatorname{sign}\left(\omega_{r}-\hat{\omega}_{r}\right) \cdot \operatorname{sign}(\sin \theta)>0
$$

From (1) and (5), the estimation error of stator current and rotor flux can be derived as shown in the following equations [24].

$$
\begin{aligned}
{\left[\begin{array}{c}
s \boldsymbol{e}_{i} \\
s \boldsymbol{e}_{\psi}
\end{array}\right] } & =F\left[\begin{array}{c}
\boldsymbol{e}_{i} \\
\boldsymbol{e}_{\psi}
\end{array}\right]+H\left[\begin{array}{c}
\hat{\boldsymbol{i}}_{s} \\
\hat{\boldsymbol{\psi}}_{s}
\end{array}\right] \\
F & =\left[\begin{array}{cc}
-\frac{1}{T_{\sigma}}-\boldsymbol{g}_{1} & \lambda L_{m}\left(\frac{1}{T_{r}}-j \omega_{r}\right) \\
\frac{L_{m}}{T_{r}}-\boldsymbol{g}_{2} & -\frac{1}{T_{r}}+j \omega_{r}
\end{array}\right] \\
H & =\left[\begin{array}{cc}
0 & -j \lambda L_{m} \triangle \omega_{r} \\
0 & j \triangle \omega_{r}
\end{array}\right]
\end{aligned}
$$

Based on (16), transfer function $N(s)$ can be derived as expressed in (19).

$$
\begin{aligned}
N(s) & =\frac{\hat{\boldsymbol{\psi}}_{r}}{\left(\boldsymbol{i}_{s}-\hat{\boldsymbol{i}}_{s}\right)}=\frac{s^{2}+\left(h_{1}+j h_{2}\right) \cdot s+d_{1}+j d_{2}}{-j \triangle \omega_{r} \cdot \lambda L_{m} \cdot s} \\
h_{1} & =\left(g_{1 r}+\frac{R_{s}}{\sigma L_{s}}+\frac{R_{r}}{\sigma L_{r}}\right) \\
h_{2} & =\left(g_{1 i}-\omega_{r}\right) \\
d_{1} & =\frac{1}{T_{r}}\left(g_{1 r}+\frac{R_{s}}{\sigma L_{s}}+\lambda L_{m} g_{2 r}\right)+\omega_{r}\left(g_{1 i}+\lambda L_{m} g_{2 i}\right) \\
d_{2} & =\frac{1}{T_{r}}\left(g_{1 i}+\lambda L_{m} g_{2 i}\right)-\omega_{r}\left(g_{1 r}+\frac{R_{s}}{\sigma L_{s}}+\lambda L_{m} g_{2 r}\right)
\end{aligned}
$$

When estimating the free-running speed, torque reference is set as zero, namely the slip frequency is zero. Hence, the output frequency of the inverter equals the estimated speed $\hat{\omega}_{r}$. According to (19) and assuming $s=j \hat{\omega}_{r}, N\left(j \hat{\omega}_{r}\right)$ can be derived as:

$$
N\left(j \hat{\omega}_{r}\right)=\frac{-\hat{\omega}_{r}^{2}+d_{1}-h_{2} \hat{\omega}_{r}+j\left(h_{1} \hat{\omega}_{r}+d_{2}\right)}{\lambda L_{m} \hat{\omega}_{r} \triangle \omega_{r}} .
$$


Futhermore, $\frac{\hat{\boldsymbol{\psi}}_{r}}{\left(\boldsymbol{i}_{s}-\hat{\boldsymbol{i}}_{s}\right)}$ can be expressed in a different way as shown in (25).

$$
\frac{\hat{\boldsymbol{\psi}}_{r}}{\left(\boldsymbol{i}_{s}-\hat{\boldsymbol{i}}_{s}\right)}=\left|\frac{\hat{\boldsymbol{\psi}}_{r}}{\boldsymbol{i}_{s}-\hat{\boldsymbol{i}}_{s}}\right| e^{j \theta}=\frac{\left|\hat{\boldsymbol{\psi}}_{r}\right|}{\left|\boldsymbol{i}_{s}-\hat{\boldsymbol{i}}_{s}\right|}(\cos \theta+j \sin \theta)
$$

Comparing (24) and (25), it can be easily found that the sign of $\sin \theta$ will be the same as the sign of imaginary part of $N\left(j \hat{\omega}_{r}\right)$. Hence, the condition (15) can be changed as:

$$
\operatorname{sign}\left(\hat{\omega}_{r}\right) \cdot \operatorname{sign}\left(h_{1} \hat{\omega}_{r}+d_{2}\right)>0
$$

If feedback gain matrix is set as zero, the estimated speed can always converge to $\omega_{r}$ only when $\hat{\omega}_{r}$ satisfies the following conditions.

$$
\left|\hat{\omega}_{r}\right|>\left(\frac{L_{r} R_{s}}{L_{r} R_{s}+R_{r} L_{s}}\right)\left|\omega_{r}\right|
$$

It is clear that if motor is rotating at a high speed, $\hat{\omega}_{r}$ can not converge to $\omega_{r}$ when $\hat{\omega}_{r}$ is initially set as zero. This problem can be solved by setting $\hat{\omega}_{r}$ to a sufficiently large value with the same sign of $\omega_{r}$, as can be seen from (27). However, if the motor can rotate at both forward and backward direction, the rotational direction must be estimated before determining whether $\hat{\omega}_{r}$ is set to a positive or a negative value. To avoid the estimation of rotational direction, the feedback gain matrix is adjusted so that $d_{2}=0$. If $d_{2}=0$, the condition (26) deduced from (24) can be simplified as:

$$
h_{1}>0
$$

By solving (28) and $d_{2}=0$, a simple gain matrix $\boldsymbol{G}$ is proposed as shown in (29).

$$
\boldsymbol{G}=\left[\begin{array}{c}
-\frac{R_{s}}{\sigma L_{s}} \\
0
\end{array}\right]
$$

With the proposed feedback gain matrix (29), the estimated speed can always converge to actual speed with any initial value. As only the feedback gain matrix is changed, it is very simple in implementation.

\section{Model Predictive Current Control}

\subsection{Field oriented control with FCS-MPC}

In rotor flux oriented reference frame, stator current can be decomposed into torque component $i_{q}$ and flux component $i_{d}$. Through the control of $i_{d}$ and $i_{q}$, flux and torque can be indirectly regulated. As $i_{d}$ and $i_{q}$ are two components of complex current vector, no weighting factor is necessary. The 


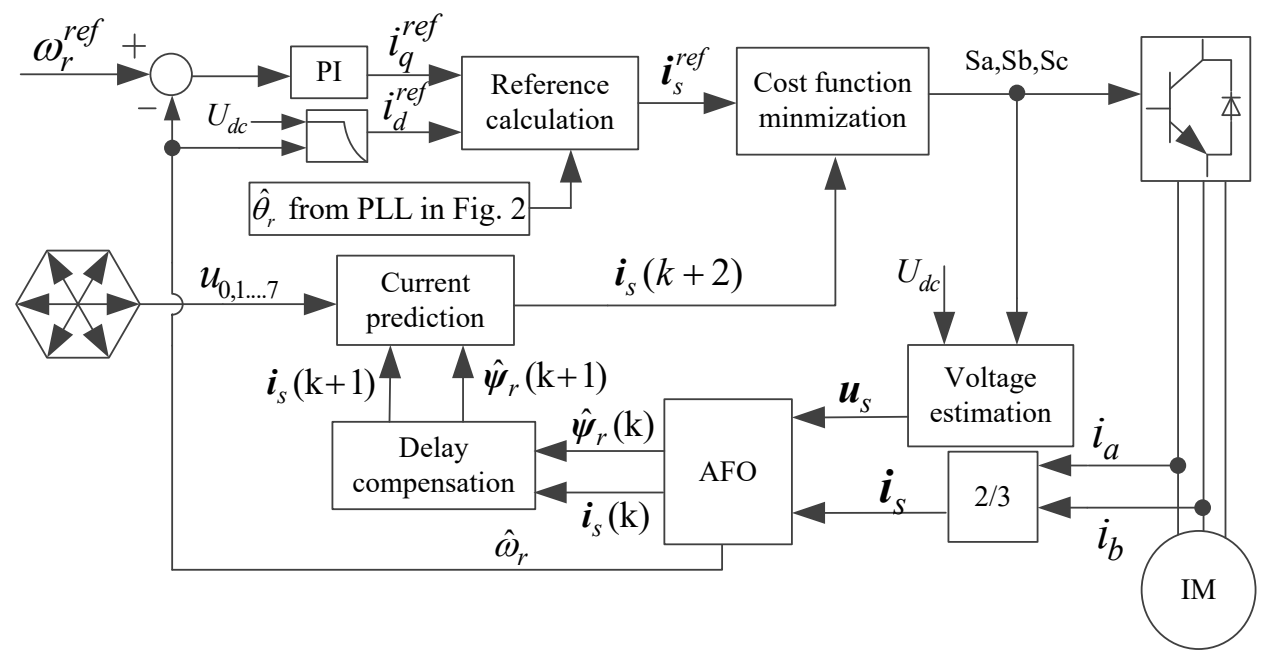

Fig. 1. Control diagram of speed sensorless FCS-MPCC

whole control diagram is shown in Fig. 1. The reference $i_{q}^{r e f}$ is generated from speed control loop using a PI controller. Rotor speed and flux are estimated based on the AFO described in Section 3. Other detailed information of the control diagram will be elaborated below.

Due to the lack of a modulator, there is no smooth output voltage reference in FCS-MPC. Hence, the estimated speed and rotor flux would contain harmonics using discrete voltage vectors. To generate a smooth rotor flux position, a phase-locked loop (PLL) with feed-forward speed is used as follows.

$$
\begin{aligned}
& \hat{\theta}_{r}=\int \omega_{e} d t \\
& \omega_{e}=\hat{\omega}_{L P F}+\omega_{s l}+\omega_{c o m}
\end{aligned}
$$

where, $\hat{\omega}_{L P F}$ is the output of a low pass filter (LPF) of $\hat{\omega}_{r}$, as shown in (32); $\omega_{s l}$ is slip frequency, which can be calculated based on $i_{d}^{r e f}$ and $i_{q}^{r e f} ; \omega_{c o m}$ is the compensating term to eliminate the phase delay in dynamic process.

$$
\begin{aligned}
\hat{\omega}_{L P F} & =\frac{\hat{\omega}_{r}}{\tau_{1} s+1} \\
\omega_{s l} & =\frac{i_{q}^{r e f}}{T_{r} \cdot i_{d}^{r e f}}
\end{aligned}
$$

When $\hat{\theta}_{r}$ is accurately calculated, $\hat{\psi}_{r q}$ would be zero. As $\hat{\omega}_{L P F}$ is the output of a low pass filter, phase delay would occur during dynamic process and thus $\hat{\psi}_{r q}$ would deviate from zero. Hence, a 


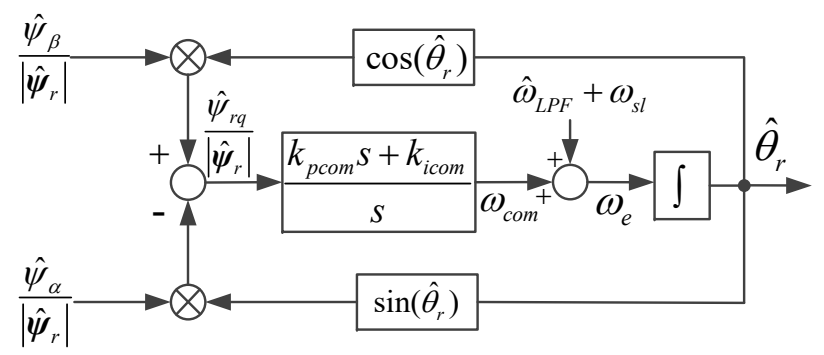

Fig. 2. Block diagram of PLL

PI controller is used to compensate phase delay as follow.

$$
\omega_{c o m}=\frac{k_{p c o m} \cdot s+k_{i c o m}}{s} \frac{\hat{\psi}_{r q}}{\hat{\psi}_{r}}
$$

where $\hat{\psi}_{r}$ is the amplitude of the rotor flux. The control diagram is shown in Fig. 2, which is actually a PLL with feed-forward speed. The transfer function of PLL can be derived as :

$$
\frac{\hat{\theta}_{r}}{\theta_{r}}=\frac{k_{p c o m} \cdot s+k_{i c o m}}{s^{2}+k_{p c o m} \cdot s+k_{i c o m}}
$$

where $\theta_{r}$ is phase angle of $\hat{\boldsymbol{\psi}}_{r}$.

The parameters of PI controller can be set as (36) and (37) if a second-order Butterworth-type filter is assumed.

$$
\begin{aligned}
& k_{\text {pcom }}=\sqrt{2} \omega_{c} \\
& k_{i c o m}=\omega_{c}^{2}
\end{aligned}
$$

For simplicity, a fixed $\omega_{c}$ could be used in practical implementation [34]. Omitting tedious deduction process, the bandwidth of the closed-loop system (35) is $2.05 \omega_{c}$. To reduce the influence of low order harmonics, $\omega_{c}$ is set as $\omega_{c}=31 \mathrm{rad} / \mathrm{s}$ in this paper.

Considering $\psi_{r}=L_{m} i_{d}$ during steady state, (2) can also be expressed as

$$
T_{e}=\frac{3}{2} n_{p} \frac{L_{m}^{2}}{L_{r}} i_{d} i_{q}
$$

It can be deduced that, for a constant torque (38), if $i_{d}=i_{q}, i_{d}^{2}+i_{q}^{2}$ reaches its minimum value. Hence, to improve the efficiency, $i_{d}^{\text {ref }}$ is set to be equal to the absolute value of $i_{q}$. As shown in (39), the filtered value of $i_{q}$ is used to reduce noise.

$$
i_{d}^{r e f}=\frac{\left|i_{q}\right|}{\tau_{2} s+1}
$$




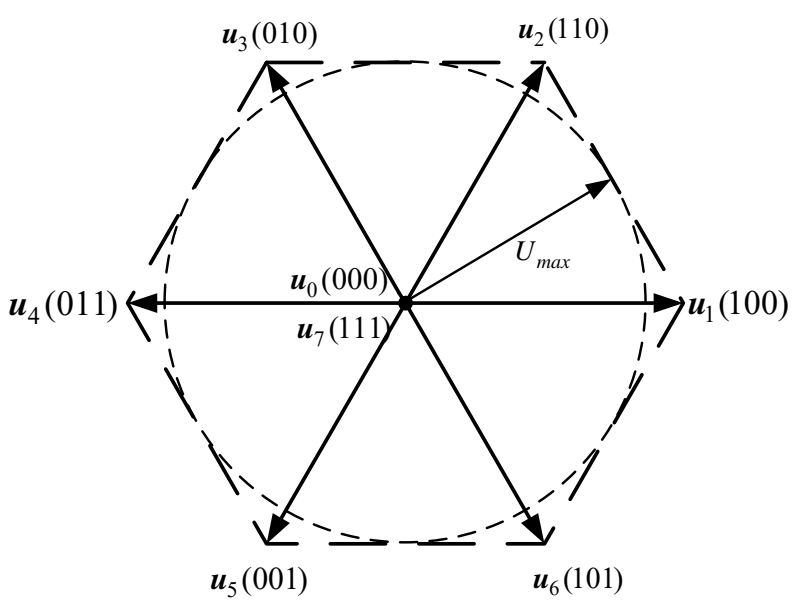

Fig. 3. Voltage vectors and corresponding switching states of a two-level inverter

To prevent saturation and keep a minimum level of flux, $i_{d}^{\text {ref }}$ need to be limited within a range in practical application, as shown in (40).

$$
i_{d}^{r e f}= \begin{cases}I_{d \min } & \text { if } i_{d}^{r e f}<I_{d \min } \\ I_{d \max } & \text { if } i_{d}^{r e f}>I_{d \max }\end{cases}
$$

To achieve field-weakening operation, $I_{d \max }$ presented in (40) should not exceed $I_{f w}$ in (41) [35].

$$
I_{f w}=\sqrt{\left(\frac{U_{\max }}{\omega_{e} L_{s}}\right)^{2}-\left(\sigma i_{q}\right)^{2}}
$$

where, $U_{\max }$ is the maximum fundamental voltage can be obtained. As there is no modulator in FCS-MPC, it is hard to calculate available $U_{\max }$ and it is set as (42) in this paper, which is the maximum radius of the circle inside the hexagon shown in Fig. 3.

$$
U_{\max }=\frac{U_{d c}}{\sqrt{3}}
$$

After obtaining $i_{d}^{r e f}$ and $i_{q}^{r e f}$, the reference of current vector can be calculated in stationary frame as

$$
\boldsymbol{i}_{s}^{r e f}=\left(i_{d}^{r e f}+j \cdot i_{q}^{r e f}\right) e^{j\left(\hat{\theta}_{r}+2 \omega_{e} T_{s c}\right)} .
$$

In (43), the phase angle of rotor flux is added by two sampling periods to compensate digital delay in practical implementation, which is normal for FCS-MPC [36]. In strict sense, $i_{d}^{\text {ref }}$ and $i_{q}^{r e f}$ at $(k+2)$ th instant are required. As the sampling frequency is much higher than the cutoff frequency of low pass filter (39) and the bandwidth of outer speed control loop, $i_{d}^{r e f}$ and $i_{q}^{r e f}$ can be approximately assumed to be constant within the prediction horizon, i.e. $i_{d q}^{r e f}(k+2) \approx i_{d q}^{r e f}(k+1) \approx$ $i_{d q}^{r e f}(k)$. After obtaining $\boldsymbol{i}_{s}^{r e f}$ based on (43), the following cost function can be used as a criterion 
to select the best voltage vector.

$$
J=\left|\boldsymbol{i}_{s}^{r e f}-\boldsymbol{i}_{s}^{k+2}\right|
$$

To evaluate cost function, the system state at $(k+1)$ th instant is first predicted based on (3) and (4) using the optimal voltage vector selected at $(k-1)$ th sampling period. Then, the stator current at $(k+2)$ th instant is predicted for each feasible voltage vectors. The voltage vector resulting in minimum $J$ is selected as optimal one.

\subsection{Starting a Rotating Motor}

In many cases, such as resuming to normal operation after short power interruption, starting a machine rotated by external load or re-powering after coasting condition of an electrical vehicle, the motor needs to be started with nonzero speed. During the estimation of free-running speed, $\omega_{s l}$ and $\omega_{\text {com }}$ in (31) is set as zero. The PI controller for speed regulation is disabled and $i_{q}^{\text {ref }}$ is

set as zero. $i_{d}^{r e f}$ is set as $I_{d \max }$ to minimize the process for building rotor flux. To respond to both forward and backward rotational direction quickly, the initial value of $\hat{\omega}_{r}$ is set as zero. As analyzed in section 3 , the estimated speed $\hat{\omega}_{r}$ can converge to actual speed $\omega_{r}$ with the proposed feedback gain matrix (29) even if there is a large initial difference. The remaining problem is to judge whether $\hat{\omega}_{r}$ is close to $\omega_{r}$ and thus the normal operation can be subsequently enabled.

When $\hat{\omega}_{r}$ approaches to $\omega_{r}, d \hat{\omega}_{r} / d t$ in (9) becomes smaller. Hence, if the filtered value of $d \hat{\omega}_{r} / d t$ is smaller than a predefined threshold, the estimation of free-running speed is terminated and normal operation is enabled. As $d \hat{\omega}_{r} / d t$ is zero before the control system is activated, a minimum time $T_{\min }$ is hold for the estimation of free-running speed to improve the reliability. The flow chart of the proposed procedure is shown in Fig. 4.

\section{Simulation and Experimental Results}

\subsection{Simulation Tests}

The proposed method is first validated in Matlab/Simulink with a two-level inverter fed IM drive. The parameters of the motor and control system are listed in Table 1. The time constants of low pass filter shown in (32) and (39) are set as $\tau_{1}=5 \mathrm{~ms}$ and $\tau_{2}=10 \mathrm{~ms}$ in the following tests respectively. The PI controller in (12) for speed estimation is chosen as $k_{p \omega}=1, k_{i \omega}=80 . i_{d}^{\text {ref }}$ is adjusted based on (39) and the limitation is set as $I_{d m i n}=2 \mathrm{~A}, I_{d \max }=6 \mathrm{~A}$. The minimum time $T_{\text {min }}$ for initial speed estimation is $0.05 \mathrm{~s}$. The filter time constant $\tau_{3}$ and threshold $X_{\min }$ in Fig. 4 are chosen as $\tau_{3}=10 \mathrm{~ms}$ and $X_{\min }=10$.

Fig. 5 shows the responses of the proposed method when the motor is started from a freerunning speed $1200 \mathrm{rpm}$. It is seen that the estimated speed $\hat{\omega}_{r}$ converges to actual speed quickly. There is no oscillation or inrush current during starting process. After $\hat{\omega}_{r}$ reaches $\omega_{r}$, the normal operation is enabled and the motor speed is accelerated to the reference speed $1500 \mathrm{rpm}$. When $i_{q}$ decreases, $i_{d}$ decreases accordingly. Hence, the efficiency can be improved during light load 


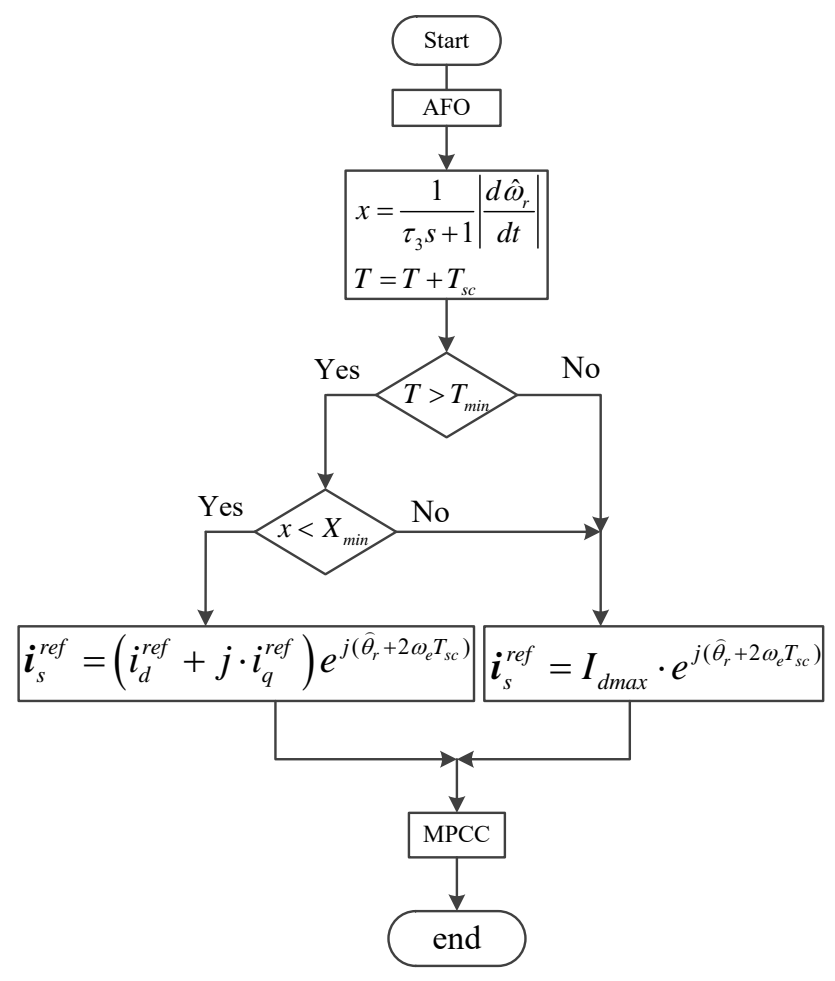

Fig. 4. Flowchart of starting procedure

Table 1 Parameters of IM and control system

\begin{tabular}{lcr}
\hline Rated power & $P_{N}$ & $2.2 \mathrm{~kW}$ \\
DC-bus voltage & $U_{d c}$ & $540 \mathrm{~V}$ \\
Rated voltage & $U_{N}$ & $380 \mathrm{~V}$ \\
Rated frequency & $f_{N}$ & $50 \mathrm{~Hz}$ \\
Number of pole pairs & $N_{p}$ & 2 \\
Stator resistance & $R_{s}$ & $1.76 \Omega$ \\
Rotor resistance & $R_{r}$ & $1.29 \Omega$ \\
Mutual inductance & $L_{m}$ & $0.158 \mathrm{H}$ \\
Stator inductance & $L_{s}$ & $0.170 \mathrm{H}$ \\
Rotor inductance & $L_{r}$ & $0.170 \mathrm{H}$ \\
Maximum stator current & $I_{\max }$ & $10 \mathrm{~A}$ \\
Control period & $T_{s c}$ & $50 \mu \mathrm{s}$ \\
\hline
\end{tabular}

conditions. From the time the control system is activated to the time the speed control loop is enabled, the whole process takes less than 0.1 second. This test confirms that a free-running motor can be smoothly and quickly start without speed sensor using the proposed method.

To illustrate the effectiveness of the proposed feedback gain matrix (29), the same tests were carried out when the gain matrix are selected as zero and $G_{1}$. With $G_{1}$, the poles of the observer 

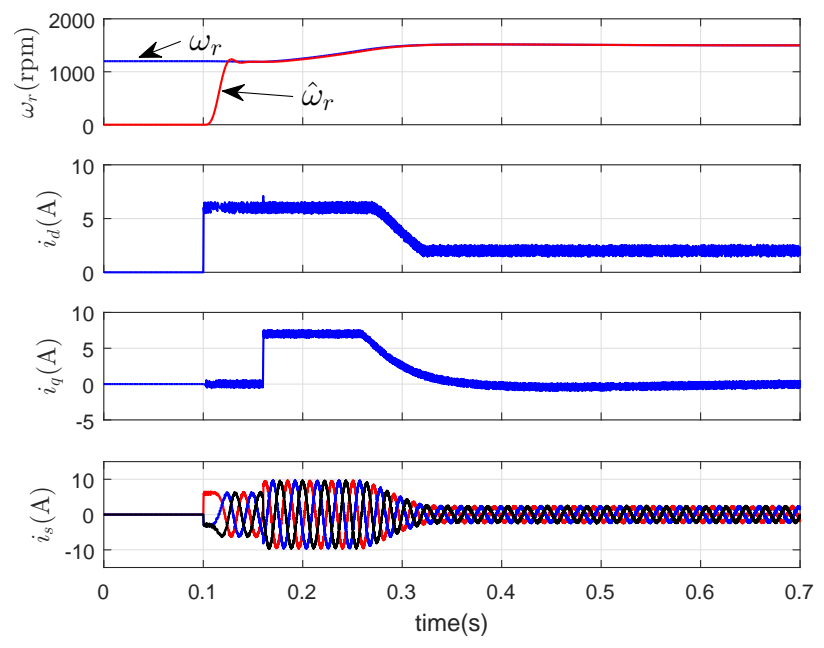

Fig. 5. Simulated responses of starting from $1200 \mathrm{rpm}$ to $1500 \mathrm{rpm}$ with the proposed method
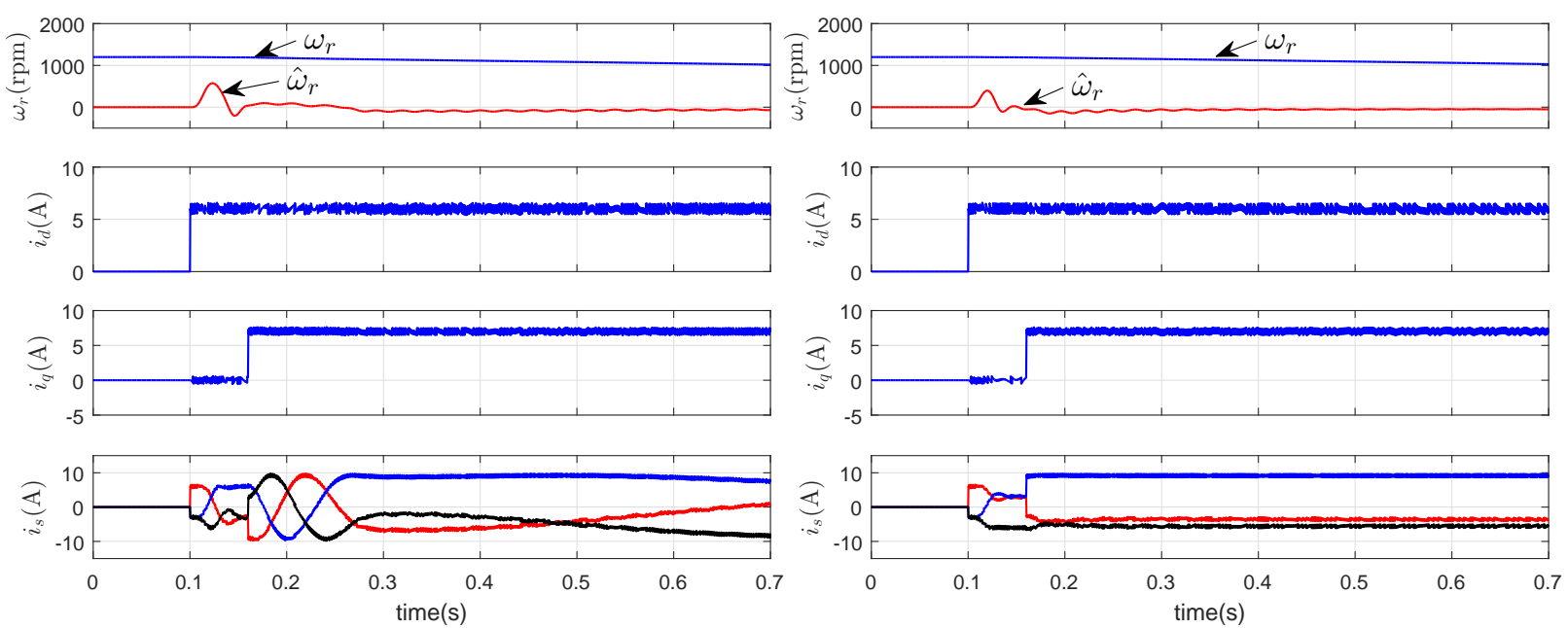

(a)

(b)

Fig. 6. Simulated responses of starting from $1200 \mathrm{rpm}$ to $1500 \mathrm{rpm}$ with (a) zero gain matrix and (b) $\boldsymbol{G}_{1}$

is proportional to the poles of IM itself [37].

$$
\begin{aligned}
\boldsymbol{G}_{1} & =\left[\begin{array}{c}
f \\
\frac{\lambda L_{r}\left(k^{2}-1\right) R_{s}-f}{\lambda L_{m}}
\end{array}\right] \\
f & =\lambda(k-1)\left(R_{s} L_{r}+R_{r} L_{s}\right)+j(1-k) \hat{\omega}_{r}
\end{aligned}
$$

where, $k$ is a proportional constant and it is set as $k=1.2$ in the test. As can be seen from Fig. 6 , the estimated speed can not converge to actual speed in both cases and start of the motor fails. Hence, the developed FCS-MPCC must work together with AFO based on gain matrix (29) to achieve smooth and quick start of a free-running motor. 


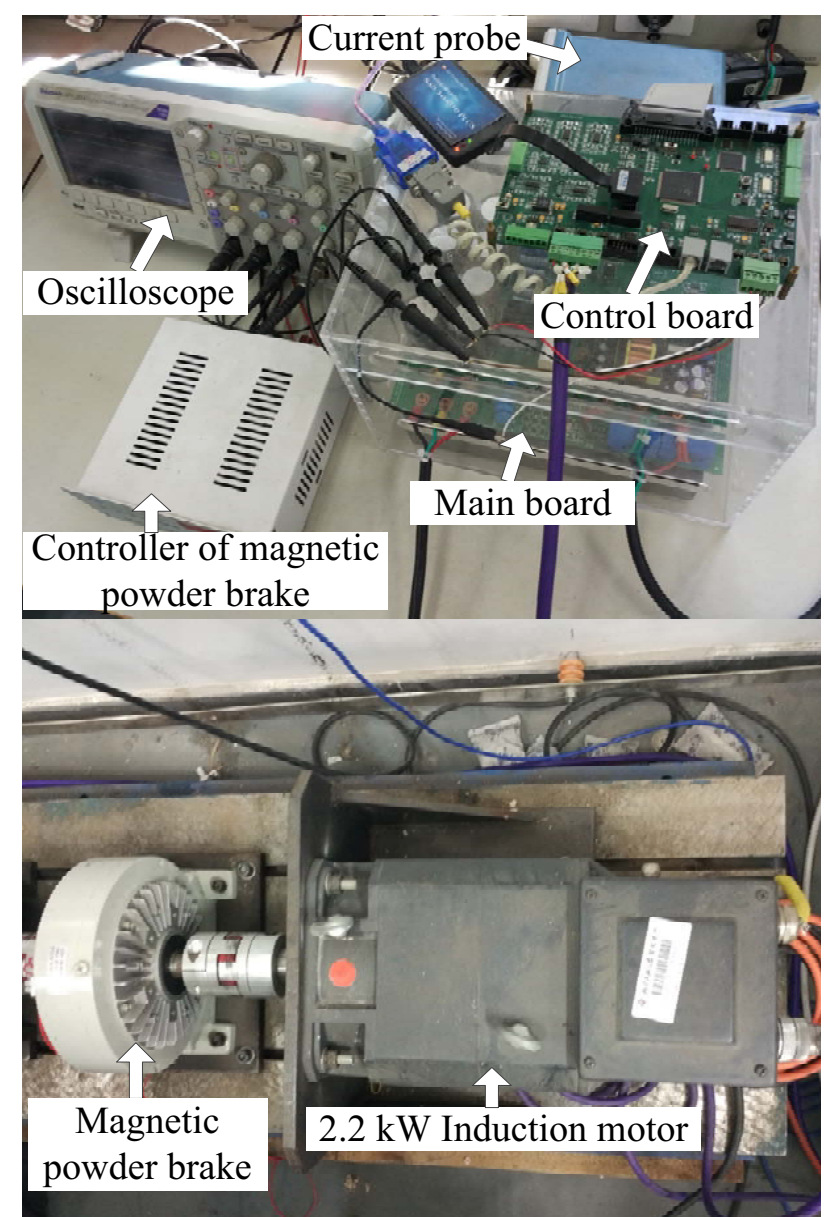

Fig. 7. Experimental setup

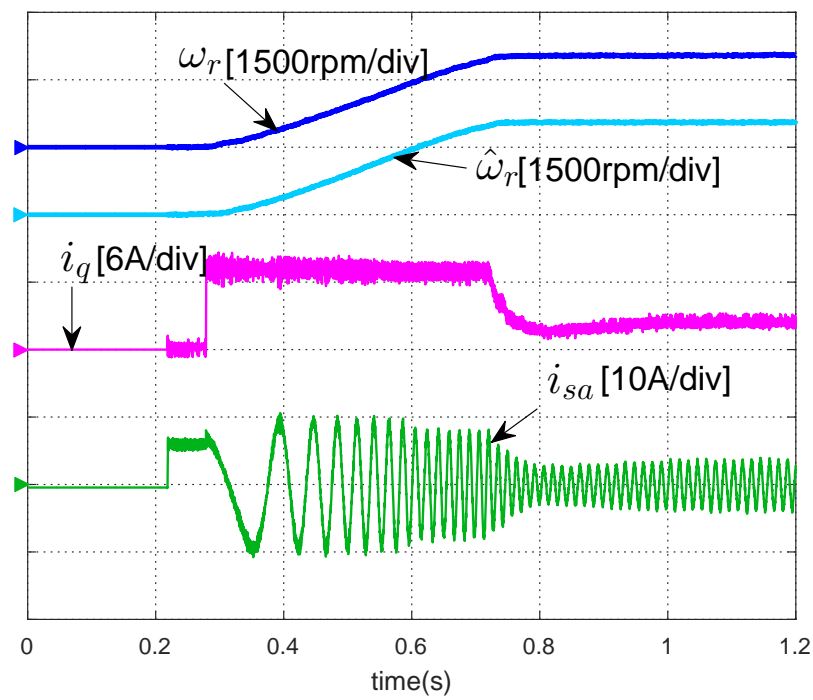

Fig. 8. Starting from standstill to $2100 \mathrm{rpm}$ with the proposed method

\subsection{Experimental Tests}

The experimental setup is shown in Fig. 7. The developed scheme is implemented on a digital control board, which was built based on a Texas Instruments TMS320F28335 digital signal processor. The deadtime for the inverter is $3 \mu \mathrm{s}$. An on-board DA converter is used to get the information of internal variables. The actual speed is measured through an incremental optical encoder, which is only displayed on an oscilloscope for comparison. The parameters used in the experimental tests are the same as those used in simulation tests.

Fig. 8 shows the responses when the machine starts from standstill to $2100 \mathrm{rpm}$ without load. The recorded curves from top to the bottom are actual speed, estimated speed, torque current $i_{q}$ and stator current. It can be seen that the motor accelerates quickly to the reference speed after initial speed estimation. The estimated speed follows the actual speed well during the whole process, indicating that the observer and controller work well over a wide speed range.

The proposed method can start the motor not only from standstill but also from a rotating state. Fig. 9 shows the results when the motor is started from free-running conditions. At the beginning, 


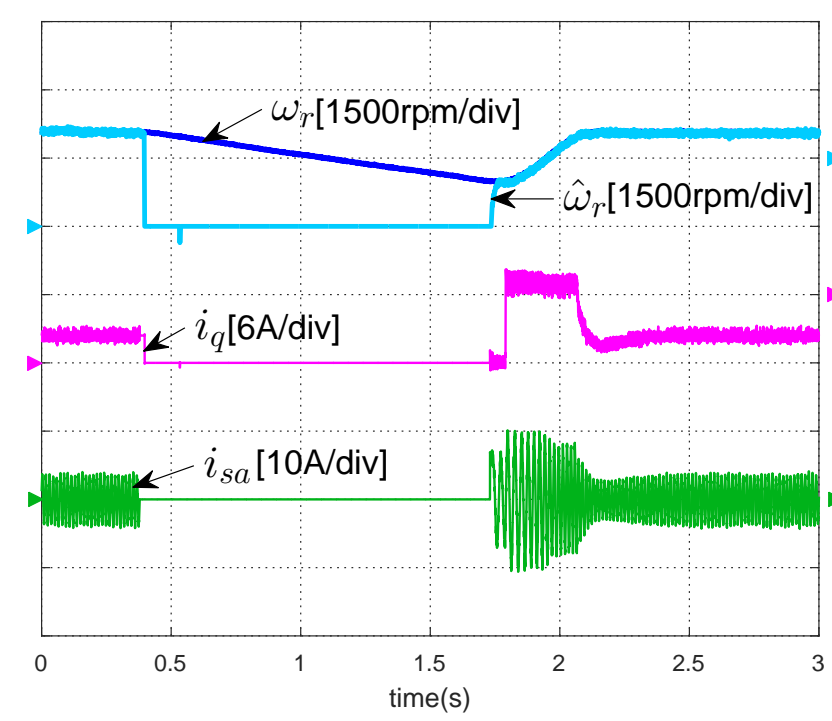

(a)

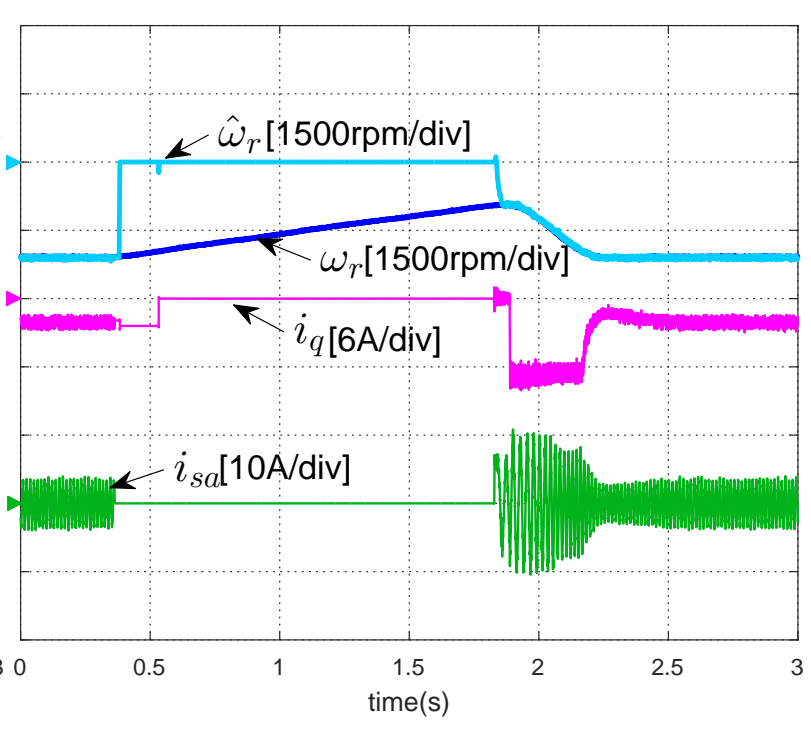

(b)

Fig. 9. Starting from free-running state with (a) forward speed and (b) backward speed based on proposed method

the motor is steadily rotating at $2100 \mathrm{rpm}$ and $-2100 \mathrm{rpm}$ as shown in Figs. 9a and 9b respectively. Then, the power is turned off and the rotor speed decreases gradually due to the friction load. After a moment, the controller is re-activated. For a direct comparison, the zero position of actual speed and estimated speed is set the same in this test. It is clear that, the estimated speed can respond for both rotational directions quickly. No obvious oscillation and inrush current can be observed during starting process. After the estimation of initial speed, the rotor speed is quickly re-accelerated to original reference. The estimated speed almost coincides with the actual speed, showing good accuracy during dynamic and steady operation.

However, if feedback gain matrix is set as zero, the estimated speed can not converge to actual speed if the motor is initially running at a high speed, as shown in Fig. 10. It is seen that when the rotor speed decreases to a smaller value which is close to estimated speed, the motor states can still be estimated and the motor can be precisely controlled again. However, if there is a large shaft inertia, it would be time consuming to wait for the motor to stop. While with the proposed method, the motor can be started without speed sensor even at a high rotating speed.

Fig. 11 shows speed reversal from $-2100 \mathrm{rpm}$ to $2100 \mathrm{rpm}$. The motor speed quickly decelerates to zero and then accelerates to the reference. The zero-crossing of speed is relatively smooth without obvious chattering. This test confirms that the observer and controller works well during dynamic processes with variable speed.

The robustness against external disturbance is also tested. The responses of actual speed, torque current $i_{q}$, flux current $i_{d}$ and stator current are plotted in Fig. 12. At the beginning, the motor is controlled at $1500 \mathrm{rpm}$ without load. After a moment, an external load is suddenly applied and then released. The rotor speed drops slightly and returns to its reference quickly when the load is 

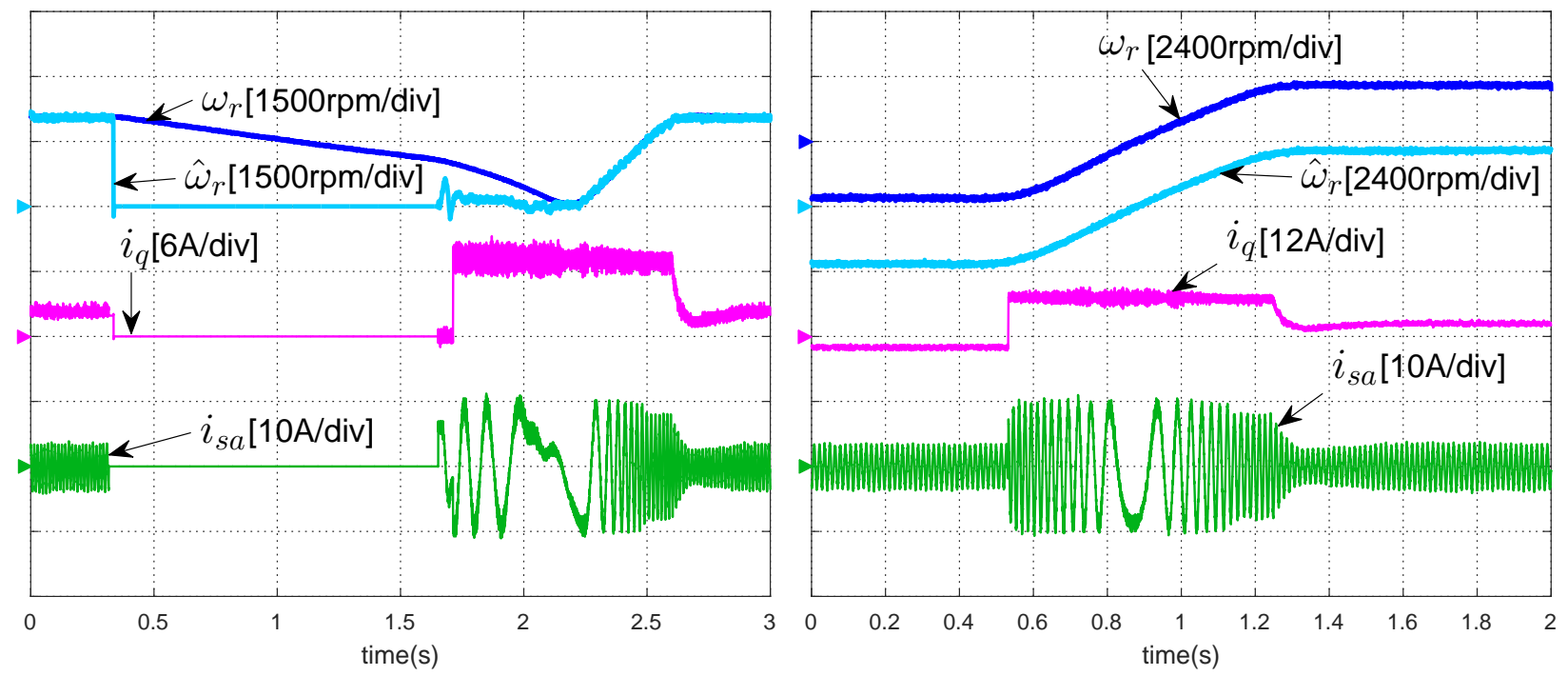

Fig. 10. Starting from a high initial speed with Fig. 11. Responses during speed reversal with zero feedback gain matrix

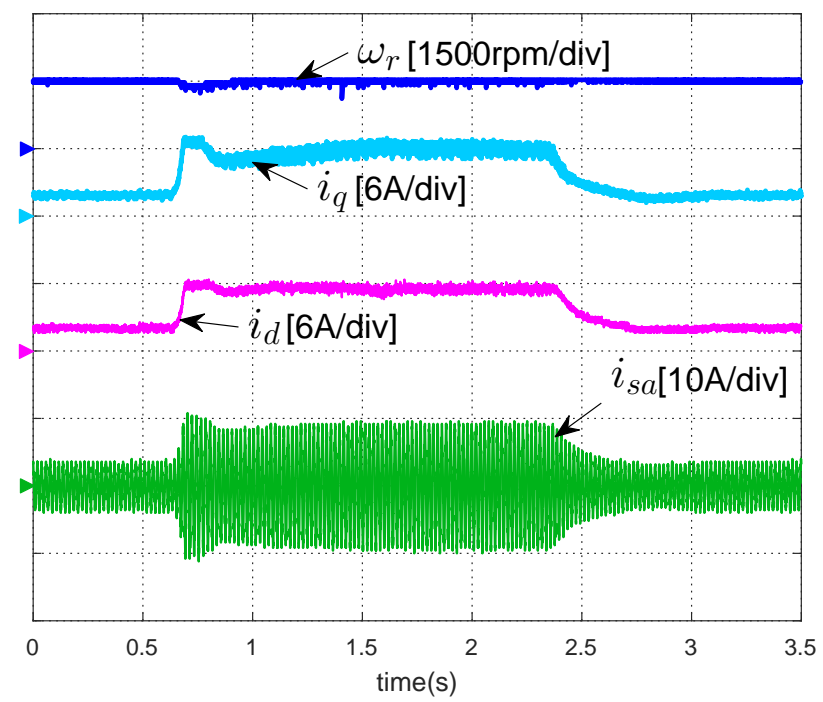

Fig. 12. Responses with sudden load change the proposed method
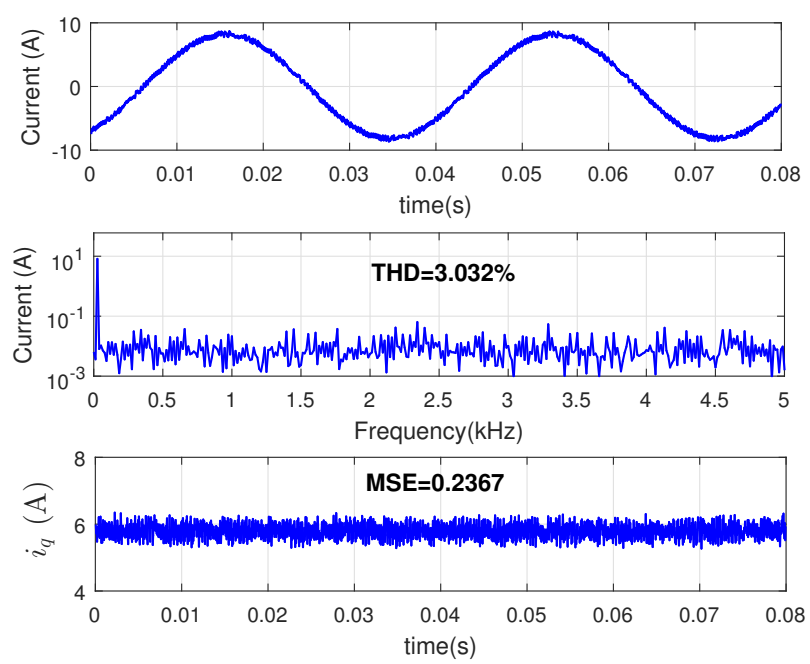

Fig. 13. Current waveform and spectrum at 750 rpm with $14 \mathrm{Nm}$ load torque

applied, showing good robustness against external load disturbance. It is also clear that $i_{d}$ varies along with $i_{q}$, as expressed in (39). When the external load decreases, $i_{d}$ is also decreased. This can help to improve efficiency during light load condition.

Fig. 13 shows steady state performance at $\omega_{r}=750 \mathrm{rpm}$ when $14 \mathrm{Nm}$ external load torque is applied. It can be seen that stator current is sinusoidal with total harmonic distortion as 3.032\%. The torque current $i_{q}$ is well controlled and its mean squared error (MSE) is $0.2367 \mathrm{~A}$. 


\section{Conclusion}

In this paper, a speed-sensorless FCS-MPCC based on AFO is proposed. The convergence condition of speed estimation is analyzed and a feedback gain matrix is proposed to make the controller capable of starting a rotating motor with unknown rotational direction and speed. Detailed implementation of FCS-MPCC combined with AFO based on the proposed feedback gain matrix is illustrated.

The developed method presents good dynamic performance against load disturbance. The responses during speed reversal and starting processes are satisfactory. Additionally, motors rotating at a high speed can be smoothly and quickly started without the use of a speed sensor. The whole control system has the merits of simplicity, low cost and fast dynamic responses, which can be easily accomplished with a proper DSP. Hence, the presented scheme is suitable for industrial applications. Simulation and experimental results on a $2.2-\mathrm{kW}$ IM drive platform confirm the effectiveness of the proposed method.

\section{References}

[1] Zhang, Y., Yang, H., Xia, B.: 'Model predictive torque control of induction motor drives with reduced torque ripple', IET Electr. Power Appl., 2015, 9, (9), pp. 595-604

[2] Rodriguez, J., Kennel, R., Espinoza, J., et al.: 'High-performance control strategies for electrical drives: An experimental assessment', IEEE Trans. Ind. Electron., 2012, 59, (2), pp. 812 $-820$

[3] Rojas, C., Rodriguez, J., Villarroel, F., et al.: 'Predictive torque and flux control without weighting factors', IEEE Trans. Ind. Electron., 2013, 60, (2), pp. $681-690$

[4] Kouro, S., Perez, M. A., Rodriguez, J., et al:: 'Model predictive control: Mpc's role in the evolution of power electronics', IEEE Ind. Electron. Mag., 2015, 9, (4), pp. 8-21

[5] Vazquez, S., Leon, J. I., Franquelo, L. G., et al.: 'Model predictive control: A review of its applications in power electronics', IEEE Ind. Electron. Mag., 2014, 8, (1), pp. 16-31

[6] Geyer, T., Quevedo, D.: 'Multistep finite control set model predictive control for power electronics', IEEE Trans. Power Electron., 2014, 29, (12), pp. 6836-6846

[7] Miranda, H., Cortes, P., Yuz, J., et al.: 'Predictive torque control of induction machines based on state-space models', IEEE Trans. Ind. Electron., 2009, 56, (6), pp. 1916-1924

[8] Zhang, Y., Xia, B., Yang, H.: 'Performance evaluation of an improved model predictive control with field oriented control as a benchmark', IET Electr. Power Appl., 2016, doi: 10.1049/iet-epa.2015.0614

[9] Niu, F., Wang, B., Babel, A. S., et al.: 'Comparative evaluation of direct torque control strategies for permanent magnet synchronous machines', IEEE Trans. Power Electron., 2016, 31, (2), pp. 1408-1424 
[10] Rodriguez, J., Cortes, P.: 'Predictive control of power converters and electrical drives' (WileyIEEE Press, 2012)

[11] Rodriguez, J., Kazmierkowski, M., Espinoza, J., et al.: 'State of the art of finite control set model predictive control in power electronics', IEEE Trans. Ind. Informat., 2013, 9, (2), pp. 1003-1016

[12] Zhang, Y., Yang, H., Xia, B.: 'Model-predictive control of induction motor drives: Torque control versus flux control', IEEE Trans. Ind. Appl., 2016, 52, (5), pp. 4050-4060

[13] Davari, S., Khaburi, D., Kennel, R.: 'An improved FCS-MPC algorithm for an induction motor with an imposed optimized weighting factor', IEEE Trans. Power Electron., 2012, 27, (3), pp. $1540-1551$

[14] Zhang, Y., Yang, H.: 'Model-predictive flux control of induction motor drives with switching instant optimization', IEEE Trans. Energy Convers., 2015, 30, (3), pp. 1113-1122

[15] Zhang, Y., Yang, H.: 'Two-vector-based model predictive torque control without weighting factors for induction motor drives', IEEE Trans. Power Electron., 2016, 31, (2), pp. 13811390

[16] Davari, S. A., Khaburi, D. A., Wang, F., et al.: 'Using full order and reduced order observers for robust sensorless predictive torque control of induction motors', IEEE Trans. Power Electron., 2012, 27, (7), pp. 3424-3433

[17] Martín, C., Arahal, M. R., Barrero, F., et al.: 'Five-Phase Induction Motor Rotor Current Observer for Finite Control Set Model Predictive Control of Stator Current', IEEE Trans. Ind. Electron., 2016, 63, (7), pp. 4527-4538

[18] Martín, C., Arahal, M. R., Barrero, F., et al.: 'Multiphase rotor current observers for current predictive control: A five-phase case study', Control Eng. Pract., 2016, 49, pp. 101-111

[19] Rodas, J., Barrero, F., Arahal, M. R., et al.: 'Online Estimation of Rotor Variables in Predictive Current Controllers: A Case Study Using Five-Phase Induction Machines', IEEE Trans. Ind. Electron., 2016, 63, (9), pp. 5348-5356

[20] Xia, C., Wang, M., Song, Z., et al.: 'Robust Model Predictive Current Control of ThreePhase Voltage Source PWM Rectifier With Online Disturbance Observation', IEEE Trans. Ind. Informat., 2012, 8, (3), pp. 459-471

[21] Wang, F., Zhang, Z., Davari, S. A., et al.: 'An encoderless predictive torque control for an induction machine with a revised prediction model and EFOSMO', IEEE Trans. Ind. Electron., 2014, 61, (12), pp. 6635-6644

[22] Zhang, Y., Zhu, J., Zhao, Z., et al.: 'An improved direct torque control for three-level inverterFed induction motor sensorless drive', IEEE Trans. Power Electron., 2012, 27, (3), pp. 1502 $-1513$

[23] Chen, B., Wang, T., Yao, W., et al.: 'Speed convergence rate-based feedback gains design of adaptive full-order observer in sensorless induction motor drives', IET Electr. Power Appl., 2014, 8, (1), pp. 13-22 
[24] Suwankawin, S., Sangwongwanich, S.: 'Design strategy of an adaptive full-order observer for speed-sensorless induction-motor drives-tracking performance and stabilization', IEEE Trans. Ind. Electron., 2005, 53, (1), pp. 96-119

[25] Zaky, M.: 'Stability Analysis of Speed and Stator Resistance Estimators for Sensorless Induction Motor Drives', IEEE Trans. Ind. Electron., 2012, 59, (2), pp. 858 -870

[26] Wang, B., Zhao, Y., Yu, Y., et al.: 'Speed-Sensorless Induction Machine Control in the FieldWeakening Region Using Discrete Speed-Adaptive Full-Order Observer', IEEE Trans. Power Electron., 2016, 31, (8), pp. 5759-5773

[27] Diao, L.-J., nan Sun, D., Dong, K., et al.: 'Optimized Design of Discrete Traction Induction Motor Model at Low-Switching Frequency', IEEE Trans. Power Electron., 2013, 28, (10), pp. 4803-4810

[28] Iura, H., Ide, K., Hanamoto, T., et al.: 'An estimation method of rotational direction and speed for free-running ac machines without speed and voltage sensor', IEEE Trans. Ind. Appl., 2011, 47, (1), pp. 153-160

[29] Kim, S., Par, H., Yoo, A., et al.: 'Soft-restarting of free-run induction motors driven by small DC-link capacitor inverters', in IEEE Energy Conversion Congr. Expo., Montreal, Canada, Sept 2015, pp. 4441-4446

[30] Habibullah, M., Lu, D. D. C.: 'A speed-sensorless FS-PTC of induction motors using extended kalman filters', IEEE Trans. Ind. Electron., 2015, 62, (11), pp. 6765-6778

[31] Wang, F., Chen, Z., Stolze, P., et al.: 'Encoderless finite-state predictive torque control for induction machine with a compensated MRAS', IEEE Trans. Ind. Informat., 2014, 10, (2), pp. 1097-1106

[32] Chapra, S. C., Canale, R. P.: 'Numerical methods for engineers' (McGraw-Hill, 6th edn. 2010)

[33] Zhang, Y., Yang, H.: 'Generalized two-vector-based model-predictive torque control of induction motor drives', IEEE Trans. Power Electron., 2015, 30, (7), pp. 3818-3829

[34] Wang, G., Li, Z., Zhang, G., et al.: 'Quadrature PLL-based high-order sliding-mode observer for IPMSM sensorless control with online MTPA control strategy', IEEE Trans. Energy Convers., 2013, 28, (1), pp. 214-224

[35] Kim, S.-H., Sul, S.-K.: 'Maximum torque control of an induction machine in the field weakening region', IEEE Trans. Ind. Appl., 1995, 31, (4), pp. 787-794

[36] Cortes, P., Rodriguez, J., Silva, C., et al.: 'Delay compensation in model predictive current control of a three-phase inverter', IEEE Trans. Ind. Electron., 2012, 59, (2), pp. 1323-1325

[37] Kubota, H., Matsuse, K.: 'Speed sensorless field-oriented control of induction motor with rotor resistance adaptation', IEEE Trans. Ind. Appl., 1994, 30, (5), pp. 1219-1224 